\title{
PRODUÇÃO DE INFORMAÇÃO SOBRE O ACESSO DOS ADOLESCENTES AOS SERVIÇOS DE SAÚDE: UMA EXPERIÊNCIA NO NÍVEL LOCAL NA VENEZUELA ${ }^{1}, 2$
}

\author{
Henny Luz Heredia Martínez ${ }^{3}$ \\ Instituto de Altos Estudios "Dr. Arnoldo Gabaldon" \\ hennyluzhm@hotmail.com
}

Elizabeth Artmann ${ }^{4}$ Escola Nacional de Saúde Pública, Fundação Oswaldo Cruz. Rio de Janeiro, RJ, Brasil bethartmann@gmail.com

\begin{abstract}
Resumo
Neste artigo pretende-se discutir a produção de informação sobre o acesso dos adolescentes aos serviços de saúde no nível local. Trata-se de um estudo de caso múltiplo, realizado em três municípios do estado Nueva Esparta. Foram incluídos 35 estabelecimentos, 35 profissionais e 246 adolescentes. Nos resultados as pontuações variam entre $10,9 \%$ e $67,2 \%$ significando necessidade de melhoria importante. A dimensão disponibilidade é limitada por fatores como: escassez de medicamentos, contraceptivos e infraestrutura. Sobre aceitabilidade, $96 \%$ dos adolescentes confirmaram que a política de confidencialidade não estava visível. Para os profissionais há necessidade de treinamento para melhorar a atenção aos adolescentes. Conclui-se que a aceitabilidade é a dimensão mais afetada, como as normas 2,4 e 8 . O processo de avaliação das normas pode ser um espaço de capacitação/reflexão sobre os principais fatores que influenciam o acesso. Os resultados do estudo permitiram mostrar um caminho para produzir evidências sobre o acesso dos adolescentes aos serviços de saúde.
\end{abstract}

Palavras-chave: Adolescente. Equidade no Acesso aos Serviços de Saúde. Serviços de Saúde. Atenção à Saúde.

\section{THE ORODUCTION OF INFORMAION ON THE ACCESS OF ADOLESCENTS TO} HEALTH SERVICES: AN EXPERIENCE AT THE LOCAL LEVEL IN VENEZUELA

\begin{abstract}
This article aims to discuss the production of information on the access of adolescents to local health services. It is a multiple case study was conducted in three municipalities of Nueva Esparta State, 35 establishments, 35 professionals and 246 adolescents were included. In the results the scores vary between 10,9\% and 67,2\% meaning that significant improvement is needed. The extent of availability is limited by factors such as drug shortages, contraceptives and infrastructure. Regarding acceptability, $96 \%$ of adolescents confirmed that the confidentiality policy was not visible. Professionals need training to improve the attention to adolescents. It is concluded that acceptability is the most affected dimension, such as standards 2, 4 and 8 . The process of evaluating standards could be a space for empowerment/reflection on the main factors that influence access. The results of the study showed the way to produce evidence on adolescents' access to health services.
\end{abstract}

Keywords: Adolescent. Equity in Access to Health Services. Health services. Health Care.

\footnotetext{
${ }^{1}$ Agradecimentos: Este trabalho foi realizado como o apoio do Instituto de Altos Estudos "Dr. Arnoldo Gabaldon" do Ministério do Poder Popular para a Saúde da Venezuela; do Programa de Estudantes-Convênio de Pós-Graduação (PEC-PG), da Capes/CNPq/MRE-Brasil e da FAPERJ.

${ }^{2}$ Henny Luz Heredia Martínez, participou no levantamento e análise dos dados; redação e revisão final do artigo; Elizabeth Artmann participou na redação e revisão final do artigo.

${ }^{3}$ Coordenação de Epidemiología do Instituto de Altos Estudios “Dr. Arnoldo Gabaldon”.

${ }^{4}$ Pesquisadora do Departamento de Administração e Planejamento em Saúde da ENSP/FIOCRUZ.
}

Esta obra está licenciada sob uma Licença Creative Commons Attribution 3.0 P2P \& INOVAÇÃO, Rio de Janeiro, v. 6 n. 1, Ed. Especial, p.101-125, 2019. 


\section{INTRODUÇÃO}

O acesso dos adolescentes aos serviços de saúde de qualidade tem sido um tema prioritário em várias iniciativas globais (INTERAGENCY TASK TEAM ON HIV; YOUNG PEOPLE, 2008; OMS, 2014; OPS; OMS, 2014). Mas persistem as desigualdades na prestação de serviços de saúde (SS) e a escassa coordenação (PATTON et al., 2014). As boas práticas se restringem a alguns países e, em geral os SS não expressam necessariamente o estabelecido nos marcos legais (CLIFTON; HERVISH, 2013; OMS, 2014).

Na América Latina e no Caribe os adolescentes são um dos grupos que menos utilizam os SS e a oferta de serviços para eles e seu acesso são limitados (WORLD HEALTH ORGANIZATION, 2017). Com frequência os adolescentes não se identificam com os serviços de cuidados primários de saúde, encontrando baixa aceitabilidade devido à percepção de falta de respeito, falta de confidencialidade e privacidade, medo de estigmatização/discriminação por parte dos profissionais de saúde, infraestruturas precárias e escassez de medicamentos e insumos (KRUK et al., 2018; OMS, 2014; WORLD HEALTH ORGANIZATION, 2017).

O conceito de acesso é complexo e multidimensional (DONABEDIAN, 1973; PENCHANSKY; THOMAS, 1981; THIEDE; AKWEONGO; DI MCLNTYRE, 2014). Para Thiede et al (2014) o acesso não se restringe ao uso dos SS, refere-se mais à "liberdade" de utilizar tais serviços e está relacionado com a capacidade do sistema de saúde de produzir serviços adaptados às necessidades da população (AITKEN; THOMAS, 2004; DONABEDIAN, 1973; OLIVER, 2004; PENCHANSKY; THOMAS, 1981; THIEDE; AKWEONGO; DI MCLNTYRE, 2014). A dinâmica entre o sistema de saúde e os indivíduos representa o potencial para se relacionar e fazer ajustes que permitam um melhor funcionamento (THIEDE; AKWEONGO; DI MCLNTYRE, 2014).

Os autores consideram três dimensões para analisar o acesso (THIEDE; AKWEONGO; DI MCLNTYRE, 2014). A disponibilidade (acesso físico) que inclui: distância entre serviços e utilizadores; opções de transporte; grau de adequação dos horários; tipo e quantidade de SS prestados, composição do equipamento de saúde, e disposição de equipamentos e fornecimentos. A viabilidade financeira (acesso financeiro) refere-se ao grau de adequação entre os custos (diretos e indiretos) da utilização dos SS e a capacidade de pagamento dos indivíduos. A aceitabilidade (percepção de usuários e prestadores) abrange a relação entre as convicções de usuários e profissionais sobre a saúde; o compromisso e a confiança entre prestador/usuário e as maneiras pelas quais os arranjos organizacionais configuram as respostas dos usuários à SS. Neste contexto, considera-se a interação entre as expectativas dos 
utilizadores em relação aos profissionais de saúde e aos estabelecimentos de saúde e a dos prestadores em relação aos utilizadores (THIEDE; AKWEONGO; DI MCLNTYRE, 2014).

Esta interação é dinâmica: tanto o sistema de saúde como os indivíduos têm o potencial para interagir e fazer ajustes que permitam um melhor funcionamento onde o acesso de alguém a alguma coisa implica uma relação (THIEDE; AKWEONGO; DI MCLNTYRE, 2014). Diferente de Donabedian (1973), estes autores consideraram a acessibilidade como um aspecto da dimensão "disponibilidade".

A "liberdade" de usar os serviços requer contar com serviços aceitáveis e economicamente viáveis, à disposição da população usuária dos sistemas de saúde; assim como com um nível de informações suficientes sobre a saúde e os sistemas de saúde no nível das comunidades (THIEDE; AKWEONGO; DI MCLNTYRE, 2014). Este ponto é fundamental, porque o processo interativo está centrado na troca de informações, pelo que a dinâmica do acesso está determinada pela qualidade da comunicação entre os atores envolvidos (AITKEN; THOMAS, 2004; THIEDE; AKWEONGO; DI MCLNTYRE, 2014).

$\mathrm{Na}$ Venezuela, os fundamentos das normas dos programas de saúde identificaram a existência de barreiras de acesso dos adolescentes aos SS, como o não cumprimento das normas de atenção em saúde sexual e reprodutiva (SSR) (MINISTERIO DEL PODER POPULAR PARA LA SALUD, 2012). O Programa de Saúde do Adolescente (PSA/MPPS) funciona com presença limitada no nível estadual; são escassos os dados que se recolhem sobre a saúde e o acesso dos adolescentes aos SS e há poucos estudos que abordem esta temática.

O objetivo deste artigo foi discutir a produção de informação sobre o acesso dos adolescentes aos serviços de saúde no nível local, a partir de um estudo de caso múltiplo realizado no estado de Nueva Esparta, Venezuela.

\section{O ACESSO NA ESTRUTURA TRIANGULAR}

A proposta de Thiede et al. (2014) considera a multidimensionalidade do acesso, questão na qual há consenso na literatura. Propõem o uso de três dimensões para agrupar os fatores que influenciam o acesso e explorá-los de forma sistemática nos diferentes níveis (do sistema de saúde e dos indivíduos ou famílias). As dimensões são interdependentes (com intersecção entre elas) e ao mesmo tempo separáveis conceitualmente, porque cada uma delas está claramente delimitada e concentra fatores estreitamente associados entre si.

A primeira dimensão é a disponibilidade, também denominada acesso físico, inclui serviços de saúde apropriados à disposição dos usuários, no local e no momento em que são 
requeridos pelos usuários. Abrange a relação entre a localização dos serviços de saúde e dos usuários destes (distância), as opções de transporte; o grau de adequação dos horários; o tipo, a quantidade e qualidade de serviços de saúde prestados; a composição de cada equipe; disponibilidade de equipamentos e suprimentos (THIEDE; AKWEONGO; DI MCLNTYRE, 2014). Esta dimensão contém os aspectos que na proposta de Donabedian (1973) se referem à estrutura. Os fatores da disponibilidade como aqui apresentada, é separada por outros autores em várias dimensões: acomodação (os horários e o sistema de marcação destes); acessibilidade (a localização geográfica dos serviços de saúde); disponibilidade (o volume e o tipo de serviços); grau de adequação ou facilidade de uso físico dos serviços de saúde (THIEDE; AKWEONGO; DI MCLNTYRE, 2014).

A viabilidade financeira ou acesso financeiro, é a segunda dimensão e se refere ao grau de adequação entre o custo da utilização dos serviços de saúde e a capacidade de pagamento dos indivíduos. Esta dimensão está vinculada ao campo do financiamento da assistência à saúde e discute os riscos financeiros do adoecimento e da utilização dos serviços de saúde, assim como a proteção das famílias e comunidades contra esses riscos (THIEDE; AKWEONGO; DI MCLNTYRE, 2014).

Nesta dimensão é importante detalhar os diferentes tipos de custos gerados ao utilizar os serviços de saúde: custos gerados ao buscar a assistência (honorários de consulta, exames diagnósticos, medicamentos); outros custos diretos (transporte e alimentação especial); custos indiretos (perda de renda ou produtividade durante o trajeto até o prestador de assistência à saúde, tempo de espera para ser atendido); capacidade individual de pagamento de cada indivíduo e a renda familiar (THIEDE; AKWEONGO; DI MCLNTYRE, 2014).

Para os autores, um ponto fundamental da viabilidade financeira é conhecer como se dá a interação dos custos dos serviços de saúde e a capacidade de pagamento das famílias, com a finalidade de evitar a geração de custos sociais com efeitos catastróficos nos grupos populacionais mais vulneráveis (THIEDE; AKWEONGO; DI MCLNTYRE, 2014).

A terceira dimensão é a aceitabilidade, entendida como "a distância social e cultural entre os sistemas de saúde e seus usuários" (THIEDE; AKWEONGO; DI MCLNTYRE, 2014, p. 146), está estreitamente relacionada com a confiança entre usuários e prestadores. A confiança, entendida como uma "ideia relacional, fundamentada no juízo ou na convicção do usuário que de que o profissional agirá em seu interesse” (GILSON, 2014, p. 166), traz aportes relevantes para compreender a aceitabilidade, na medida em que vários estudos mostram sua influência no comportamento de busca por assistência à saúde (THIEDE; AKWEONGO; DI MCLNTYRE, 2014). 
Para Gilson (2014), a aceitabilidade abrange três componentes. O primeiro é a adequação entre as convicções leigas e profissionais sobre a saúde, o entendimento leigo sobre os serviços de saúde e o tratamento influência a decisão sobre se buscar ou não atendimento e em que lugar (GILSON, 2014). Alguns usuários podem sentir desconfiança quando suas práticas ou crenças sobre a saúde são diferentes ao preconizado no modelo biomédico predominante nos serviços. A confiança dos usuários nos profissionais igualmente é influenciada pela capacidade técnica (percebida) dos prestadores, como pela disponibilidade de medicamentos, insumos e equipamentos, elementos estes das garantias institucionais de competência (GILSON, 2014). Contudo, esta autora assinala que os julgamentos são dinâmicos e dependem também das experiências passadas de atendimento, das percepções sobre a adequação do cuidado e a melhora ou piora após o atendimento.

O engajamento e diálogo entre usuário e prestador, é o segundo componente da aceitabilidade, a natureza desse diálogo depende tanto das práticas comunicativas do prestador e sua atitude com o usuário, como da disposição e atitudes dos usuários para formular o diálogo com os profissionais. Assim, as práticas dos prestadores podem reforçar as percepções negativas dos usuários, e, portanto, a desconfiança. O poder dos prestadores pode ser exercido através de suas práticas comunicativas, da preservação ou não do sigilo, da demonstração de discriminação, imparcialidade e preconceito com certo grupo de usuários (GILSON, 2014).

É frequente que os profissionais de saúde, às vezes sem querer, estereotipem certos grupos de usuários (como os adolescentes), fazendo suposições a seu respeito, o que pode resultar em atenção diferente em comparação com outros grupos. Isto poderia causar desconfiança nestes usuários em relação aos prestadores (GILSON, 2014).

A influência dos arranjos organizacionais dos serviços de saúde nas reações dos usuários é o último componente da aceitabilidade. A forma como se organizam os serviços e as repostas aos usuários moldam o acesso destes. Para Gilson (2014) tais arranjos fornecem sinais aos usuários de que os prestadores agirão ou não ao seu interesse. Assim, a diversidade de serviços oferecidos, a capacidade de ser encaminhado e ter respostas às necessidades e demandas dos pacientes, podem fortalecer ou limitar a confiança dos usuários nos prestadores.

Em alguns contextos, os mecanismos de pagamento, podem gerar desconfiança nos usuários, na medida em que percebam que os prestadores estão buscando o lucro, ao invés de atender suas necessidades (GILSON, 2014). A continuidade do vínculo através das interações repetidas, como o tempo razoável com o usuário, também influencia a confiança do usuário, segundo esta autora. 
Outro dos aspectos considerados na aceitabilidade é a responsividade, relevante para o desempenho da assistência à saúde. Para Gostin \& Hodge (2003) a responsividade está conformada por oito domínios, dos quais sete são contemplados no segundo e terceiro componente da aceitabilidade:

(1) o respeito pela dignidade das pessoas; (2) autonomia para participar em decisões relacionadas à saúde; (3) confidencialidade; (4) pronta atenção; (5) qualidade adequada de cuidados; (6) comunicação; (7) acesso às redes de apoio social; e (8) escolha dos prestadores de cuidados de saúde (p. 3).

Cada um dos domínios da responsividade está apoiado em um ou mais princípios dos direitos humanos e devem considerados pelos sistemas de saúde e seus prestadores para garantir o direito ao acesso aos serviços de saúde dos usuários. Em outras palavras, a responsividade permite conhecer a experiência dos usuários dos serviços de saúde e como seus direitos são observados no acesso e na utilização dos serviços (GOSTIN; HODGE, 2003; VAITSMAN; ANDRADE, 2005).

O acesso é definido como "a interação entre os fatores do sistema de saúde e os fatores individuais ou familiares em cada dimensão" (THIEDE; AKWEONGO; DI MCLNTYRE, 2014, p. 142). Portanto, as melhorias no acesso, para estes autores, dependem da interação comunicativa entre o sistema de saúde e seus usuários A partir do modelo conceitual da estrutura triangular do acesso são examinados os fatores que o influenciam (THIEDE; AKWEONGO; DI MCLNTYRE, 2014).

Para facilitar o grau de adequação dos serviços de saúde e os fatores de acesso individuais ou comunitários, a informação de boa qualidade é necessária e transversal em todas as dimensões. A interação comunicativa entre os atores dos sistemas de saúde e os usuários/famílias exige um diálogo na base do respeito e da confiança, com envolvimento das partes. Este processo contribui no fortalecimento de comunidades capacitadas e bem informadas (THIEDE; AKWEONGO; DI MCLNTYRE, 2014). O acesso será equitativo na medida em que exista um entendimento comum entre o sistema de saúde e os fatores de acesso do indivíduo, a família ou a comunidade; e uma forma de comunicação que reconheça reciprocamente as perspectivas das partes envolvidas.

A produção de evidências sobre o acesso aos serviços de saúde exige a captação, processamento e análises de fatores/indicadores relacionadas a cada uma das três dimensões propostas no modelo tridimensional. Desta forma, a avaliação do acesso aos serviços de saúde requer pesquisas que considerem o maior número de dimensões (DONABEDIAN, 1973, 2001; THIEDE; AKWEONGO; DI MCLNTYRE, 2014), onde além de serem analisadas as dimensões mais reconhecidas nos debates políticos sobre a equidade na assistência à saúde 
(disponibilidade e a viabilidade financeira), seja incluída a aceitabilidade. É nesta última dimensão que se considera a natureza socializada dos serviços de saúde e onde são identificadas como "as relações estruturais e de poder, influenciam o comportamento e a interação de usuários e prestadores... as barreiras de aceitabilidade mostram como a desigualdade social impregna os sistemas de saúde" (GILSON, 2014, p. 185).

\section{DESENHO DO ESTUDO}

Trata-se de um estudo de caso múltiplo, realizado no estado Nueva Esparta, selecionado por ser uma das entidades federais com todas as áreas de responsabilidade delimitadas e as equipes básicas de saúde (EBS) organizados, mas não completos. O estudo é composto por três municípios/casos: Mariño, por ser o que tem melhor acesso geográfico e onde se concentra $20 \%$ da população (INSTITUTO NACIONAL DE ESTADÍSTICA, 2018) e 17\% dos estabelecimentos de saúde; Tubores e Península de Macanao, por serem os que compõem a Área de Saúde Integral Comunitária (ASIC) de mais difícil acesso geográfico (quadro 1) (HEREDIA; ARTMANN, 2018).

Para explorar cada dimensão do acesso optamos por utilizar uma parte do conjunto integral de instrumentos desenvolvidos pela Organização Pan-Americana da Saúde (OPAS)/Organização Mundial da Saúde (OMS) (2016a) específicos para avaliar a qualidade e cobertura dos serviços de saúde destinados aos adolescentes. Os instrumentos oferecem uma possibilidade de um olhar abrangente, ao incluir na análise as diferentes perspectivas: observação direta nos estabelecimentos de saúde; a opinião dos profissionais da saúde e dos adolescentes. Os critérios avaliados nos instrumentos permitiram explorar em profundidade duas das três dimensões do acesso propostas na estrutura triangular de Thiede et al. (2014) (disponibilidade e aceitabilidade).

Foram utilizados três dos nove instrumentos desenvolvidos pela OPS/OMS (2016a) para avaliar os progressos realizados na aplicação das normas mundiais para melhorar a qualidade dos serviços de saúde dos adolescentes: 1) ferramenta de observação e lista de verificação para o inventário dos estabelecimentos; 2) ferramenta de entrevista aos prestadores de cuidados de saúde e 3) ferramenta de entrevista aos adolescentes na comunidade, estas duas últimas feitas com questionários semiestruturados (OMS, 2016a, 2016b, 2016c, 2016d). Estes três instrumentos têm sido os mais utilizados em outros países da AL (GOBIERNO DE CHILE; MINISTERIO DE SALUD, 2017; OMS; OPS; MINISTERIO DE SALUD, PRESIDENCIA DE LA NACIÓN, 2009), o que mostra sua validade internacional e potencial de uso em vários 
contextos. Os instrumentos também permitem estabelecer uma linha base para iniciar os processos de melhoria dos SS e/ou fazer supervisões de apoio (OPS; OMS, 2016b).

Dos 79 critérios avaliados pelos 9 instrumentos, foram excluídos 4, que dependiam apenas da aplicação dos instrumentos que não foram utilizados (critérios 12, 33, 62 e 71 quadro 2). A partir da estrutura conceitual de Thiede et al (2014), a cada critério foi atribuída uma dimensão do acesso. Os aspectos de aceitabilidade são trabalhados em 38 critérios, os de disponibilidade em 35 e os de viabilidade financeira, apenas em 2 critérios (quadro 2).

Antes da aplicação dos instrumentos foram realizadas duas reuniões de trabalho com atores-chave do MPPS e da Direção Estadual de Saúde, para rever os instrumentos e adaptá-los aos critérios da Norma Oficial para a Atenção Integral em Saúde Sexual e Reprodutiva da Venezuela (MINISTERIO DEL PODER POPULAR PARA LA SALUD, 2013). Também foram feitas pequenas alterações na redação de algumas perguntas. Posteriormente, foi realizado um teste piloto para avaliar os instrumentos em uma amostra de três estabelecimentos de saúde, 12 adolescentes e 6 profissionais de saúde. Os dados do teste piloto não foram incluídos no estudo. Ao concluir o teste piloto os 3 instrumentos foram ajustados.

Dadas as restrições próprias do contexto venezuelano durante o ano da execução do estudo, bem como as limitações de tempo e de financiamento da pesquisa, optamos por realizar uma amostragem por conveniência (não probabilística) (PEREIRA, 2008), estabelecendo um quantitativo de $35(50 \%)$ dos estabelecimentos de saúde dos três municípios. Seguindo as recomendações da OMS/OPAS (OMS; OPS; MINISTERIO DE SALUD, PRESIDENCIA DE LA NACIÓN, 2009) foram entrevistados seis adolescentes da área de responsabilidade de cada estabelecimento de saúde e um profissional de saúde (quadro 2). Os estabelecimentos foram selecionados priorizando os que contavam com EBS com pelo menos seis meses trabalhando. Selecionados os estabelecimentos, convidou-se a participar um dos membros do EBS, de preferência o encarregado da atenção de usuários adolescentes.

Os adolescentes foram selecionados aleatoriamente, a partir do censo/ficha familiar de usuários por área de responsabilidade ou por convocatória direta nas casas (VENEZUELA; DIRECCIÓN ESTADAL DE SALUD DEL ESTADO NUEVA ESPARTA, 2017; VENEZUELA; MINISTERIO DEL PODER POPULAR PARA LA SALUD, 2017). Dos seis adolescentes, três deveriam ter usado os SS nos últimos 6 meses e os outros três não; em ambos os subgrupos foi garantida a participação do sexo feminino e masculino. Se o adolescente e/ou o pai/representante se não concordarem em participar, outro adolescente era selecionado. No total, foram incluídos 35 estabelecimentos de saúde, 35 profissionais e 246 adolescentes (em alguns estabelecimentos foi possível entrevistar 8 adolescentes) (quadro 1). 
Os instrumentos foram aplicados entre setembro e dezembro de 2017. Para a análise dos dados foi construído um banco de dados no Microsoft Excel, utilizando o sistema de qualificação baseado em pontos proposto pela OPS/OMS (OPS; OMS, 2016d), no qual são atribuídas pontuações baixas (0) às respostas ou elementos que indiquem menor desempenho e altas pontuações (1) às respostas ou elementos que indiquem um melhor desempenho. Para modelar o valor relativo da observação em comparação com as fontes de dados utilizadas (3 instrumentos), seguiu-se a recomendação da OPS/OMS (OPS; OMS, 2016c, 2016d) de aplicar um critério de ponderação. A notação ponderada por norma e por dimensão foi calculada com base nas pontuações totais de todos os critérios de uma norma ou dimensão, com o objetivo de obter a média global da norma ou dimensão. Foi utilizada a escala proposta pela OPS/OMS (OPS; OMS, 2016c) para avaliar o nível de aplicação das normas: Pontuação 10\% (Falta de cumprimento das normas); Pontuação >10\%- 40\% (Necessidade de uma melhoria importante); Pontuação >40\%- 80\% (Necessidade de alguma melhoria; Pontuação >80\% (Cumprimento das normas).

O estudo seguiu os princípios éticos da investigação com seres humanos. Foi aprovado pelo Comitê de Ética da Diretoria de Pesquisa do Instituto de Altos Estudos "Dr. Arnalddo Gabaldon" do Ministério do Poder Popular para a Saúde (MPPS) da Venezuela, em 03/04/17 sob o número 003/2017, como pela Direção Estadual de Saúde do estado Nueva Esparta em 12/04/17. O consentimento livre e informado foi obtido de cada adolescente, mediante autorização prévia dos pais/representantes, bem como dos profissionais de saúde que aceitaram participar da pesquisa.

Quadro 1 - Critérios de seleção e características dos municípios/caso, Nueva Esparta, Venezuela, 2017. Ano 2017.

\begin{tabular}{|l|c|c|c|c|}
\hline \multicolumn{1}{|c|}{$\begin{array}{c}\text { Critérios/ } \\
\text { Características }\end{array}$} & $\begin{array}{c}\text { Estado Nueva } \\
\text { Esparta }\end{array}$ & Município Mariño & Município Tubores & $\begin{array}{c}\text { Município } \\
\text { Península de } \\
\text { Macanao }\end{array}$ \\
\hline População & 584.873 & $\begin{array}{c}119.653 \\
21 \% \text { da população } \\
\text { do estado) }\end{array}$ & $\begin{array}{c}43.187 \text { (7\% da } \\
\text { população do estado) }\end{array}$ & $\begin{array}{c}30.848 \\
(5 \% \text { da população } \\
\text { do estado })\end{array}$ \\
\hline $\begin{array}{l}\text { Número de } \\
\text { municípios do estado }\end{array}$ & 11 & N/A & N/A & N/A \\
\hline $\begin{array}{l}\text { Número de Áreas de } \\
\text { Saúde Integral } \\
\text { Comunitárias (ASIC) }\end{array}$ & 7 & 1 & $\begin{array}{c}\text { Os dois municípios conformam a } \\
\text { ASIC 1- María Guevara }\end{array}$ \\
\hline $\begin{array}{l}\text { População } \\
\text { adolescente } \\
(10-19 \text { anos) }\end{array}$ & $\begin{array}{c}\text { ASIC 3- Guaiqueri } \\
\text { população do } \\
\text { estado) }\end{array}$ & $\begin{array}{c}\text { população do } \\
\text { município). }\end{array}$ & $\begin{array}{c}7.454 \\
(17 \% \text { da população } \\
\text { do município). }\end{array}$ & $\begin{array}{l}\text { População } \\
\text { adolescente } \\
(10-19 \text { anos })\end{array}$ \\
\hline $\begin{array}{l}\text { Cobertura do } \\
\text { programa de SSR na } \\
\text { população } \\
\text { adolescente (\%) }\end{array}$ & 7 & 7,3 & 5,3 & 6,2 \\
\hline
\end{tabular}




\begin{tabular}{|c|c|c|c|c|}
\hline $\begin{array}{c}\text { Critérios/ } \\
\text { Características }\end{array}$ & $\begin{array}{l}\text { Estado Nueva } \\
\text { Esparta }\end{array}$ & Município Mariño & Município Tubores & $\begin{array}{c}\text { Município } \\
\text { Península de } \\
\text { Macanao } \\
\end{array}$ \\
\hline $\begin{array}{l}\text { Adolescentes } \\
\text { grávidas (Segundo o } \\
\text { Programa de Atenção } \\
\text { Integral de Saúde } \\
\text { Sexual e } \\
\text { Reprodutiva, } 2015 \text { - } \\
\% \text { ) }\end{array}$ & 10 & 11 & 12 & 11 \\
\hline $\begin{array}{l}\text { Número } \\
\text { estabelecimentos de } \\
\text { saúde (primeiro, } \\
\text { segundo e terceiro } \\
\text { nível de } \\
\text { atendimento) }^{1}\end{array}$ & 204 & $\begin{array}{c}35 \\
(17 \% \text { dos } \\
\text { estabelecimentos do } \\
\text { estado }\end{array}$ & $\begin{array}{c}21 \\
(10 \% \text { dos } \\
\text { estabelecimentos do } \\
\text { estado }\end{array}$ & $\begin{array}{c}12 \\
(6 \% \text { dos } \\
\text { estabelecimentos do } \\
\text { estado }\end{array}$ \\
\hline $\begin{array}{l}\text { Número } \\
\text { estabelecimentos de } \\
\text { saúde selecionados } 1\end{array}$ & 35 & $\begin{array}{c}18 \\
(51 \% \text { dos } \\
\text { estabelecimentos do } \\
\text { município }\end{array}$ & $\begin{array}{c}11 \\
(52 \% \text { dos } \\
\text { estabelecimentos do } \\
\text { município }\end{array}$ & $\begin{array}{c}6 \\
(50 \% \text { dos } \\
\text { estabelecimentos do } \\
\text { município }\end{array}$ \\
\hline $\begin{array}{l}\text { Equipes de gestão } \\
\text { conformadas nas } \\
\text { ASICs dos } \\
\text { municípios }\end{array}$ & N/A & $\begin{array}{c}\text { A ASIC tem equipe } \\
\text { de gestão } \\
\text { conformada }\end{array}$ & $\begin{array}{l}\text { A ASIC tem equipe } \\
\text { de gestão conformada }\end{array}$ & $\begin{array}{c}\text { A ASIC tem equipe } \\
\text { de gestão } \\
\text { conformada }\end{array}$ \\
\hline $\begin{array}{l}\text { Censo atualizado de } \\
\text { cada estabelecimento } \\
\text { de saúde, com } \\
\text { população por área de } \\
\text { responsabilidade }\end{array}$ & Sim & Sim & Sim & Sim \\
\hline $\begin{array}{l}\text { Avanços no Sistema } \\
\text { de Informação em } \\
\text { Saúde }\end{array}$ & \multicolumn{4}{|c|}{$\begin{array}{l}\text { É uma das entidades federais mais avançadas em matéria de Sistemas de Informação } \\
\text { em Saúde. O banco de dados de natalidade é atualizado diariamente e se gera um } \\
\text { relatório mensal e um anuário estatístico anal. No ano } 2016 \text { iniciou-se neste estado a } \\
\text { prova piloto para a carga nominal da morbidade diária de todos os estabelecimentos } \\
\text { públicos de saúde. }\end{array}$} \\
\hline
\end{tabular}

Fonte: INE (INSTITUTO NACIONAL DE ESTADÍSTICA, 2018) e do Sistema de Informação do MPPS e da Direção Estadual de Saúde do estado Nueva Esparta (VENEZUELA; DIRECCIÓN ESTADAL DE SALUD DEL ESTADO NUEVA ESPARTA, 2017; VENEZUELA; MINISTERIO DEL PODER POPULAR PARA LA SALUD, 2017).

${ }^{1}$ Dados a dezembro 2016 - N/A: Não se aplica 
Quadro 2. Normas mundiais para melhorar a qualidade dos serviços de saúde para adolescentes, OPAS/OMS, 2016

\begin{tabular}{|c|c|c|c|c|c|c|c|c|}
\hline Características & Definição da Norma & Critérios & $\begin{array}{c}\mathbf{N}^{\circ} \\
\text { Critérios } \\
\text { Totais }\end{array}$ & $\begin{array}{c}\mathbf{N}^{\circ} \\
\text { Critérios } \\
\text { Avaliados }\end{array}$ & $\begin{array}{c}\mathbf{N}^{\circ} \\
\text { Critérios } \\
\text { Excluídos }\end{array}$ & $\begin{array}{c}\mathbf{N}^{\circ} \\
\text { Critérios } \\
\text { Disp. }\end{array}$ & $\begin{array}{c}\mathbf{N}^{\circ} \\
\text { Critérios } \\
\text { Acep. }\end{array}$ & $\begin{array}{c}\mathbf{N}^{\circ} \\
\text { Critérios } \\
\text { Viab. }\end{array}$ \\
\hline \begin{tabular}{lr}
\multicolumn{2}{l}{ Conhecimentos } \\
básicos sobre \\
saúde r dos \\
adolescentes
\end{tabular} & $\begin{array}{l}\text { Norma 1. O estabelecimento de saúde põe em funcionamento } \\
\text { sistemas destinados a lograr que os adolescentes estejam bem } \\
\text { informados sobre sua própria saúde e saibam onde e quando } \\
\text { obter os serviços de saúde }\end{array}$ & $1-9$ & 9 & 9 & 0 & 6 & 3 & 0 \\
\hline $\begin{array}{l}\text { Apoio } \\
\text { comunitário }\end{array}$ & $\begin{array}{l}\text { Norma 2. O estabelecimento de saúde põe em funcionamento } \\
\text { sistemas destinados a lograr que os pais, tutores e outros } \\
\text { membros da comunidade e das organizações comunitárias } \\
\text { reconheçam a importância de prestar serviços de atenção de } \\
\text { saúde aos adolescentes, respaldem esta prestação e fomentem } \\
\text { a utilização dos serviços por parte dos adolescentes }\end{array}$ & $10-17$ & 8 & 7 & 1 & 2 & 5 & 0 \\
\hline $\begin{array}{ll}\text { Conjunto } & \text { de } \\
\text { serviços } & \\
\text { apropriados } & \end{array}$ & $\begin{array}{l}\text { Norma 3. O estabelecimento de saúde oferece um conjunto de } \\
\text { serviços de informação, orientação, diagnóstico, tratamento e } \\
\text { atenção que satisfaze as necessidades de todos adolescentes. } \\
\text { Os serviços se prestam no estabelecimento e através das redes } \\
\text { de derivação de pacientes e as atividades de extensão* }\end{array}$ & $18-23$ & 6 & 6 & 0 & 6 & 0 & 0 \\
\hline $\begin{array}{l}\text { Competência dos } \\
\text { provedores de } \\
\text { atenção de saúde }\end{array}$ & $\begin{array}{l}\text { Norma 4. Os provedores de atenção de saúde possuem as } \\
\text { competências técnicas necessárias para oferecer uma prestação } \\
\text { eficaz de serviços de saúde aos adolescentes. Os profissionais } \\
\text { de saúde e também o pessoal de apoio respeitam, protegem e } \\
\text { fazem efetivos os direitos dos adolescentes à informação, à } \\
\text { privacidade, à confidencialidade, à não discriminaçãa, a uma } \\
\text { atitude isenta de prejuízos e ao respeito. }\end{array}$ & $24-35$ & 12 & 11 & 1 & 5 & 6 & 0 \\
\hline $\begin{array}{l}\text { Características } \\
\text { dos } \\
\text { estabelecimentos }\end{array}$ & $\begin{array}{l}\text { Norma 5. O estabelecimento de saúde oferece um horário de } \\
\text { atenção conveniente, um ambiente acolhedor e limpo e } \\
\text { preserva a privacidade e a confidencialidade. O centro conta } \\
\text { com os equipamentos, os medicamentos, os subministros e a } \\
\text { tecnologia necessários para garantir uma prestação eficaz de } \\
\text { serviços aos adolescentes. }\end{array}$ & $36-48$ & 13 & 13 & 0 & 10 & 3 & 0 \\
\hline $\begin{array}{l}\text { Equidade e não } \\
\text { discriminação }\end{array}$ & $\begin{array}{l}\text { Norma 6. O estabelecimento de saúde presta serviços de boa } \\
\text { qualidade a todos os adolescentes, independentemente de sua } \\
\text { capacidade de pagamento, sua idade, sexo, estado civil, grau } \\
\text { de instrução, origem étnico, orientação sexual e outras } \\
\text { características. }\end{array}$ & $49-58$ & 10 & 10 & 0 & 1 & 7 & 2 \\
\hline
\end{tabular}


ARTIGO

INOVAÇÃO

\begin{tabular}{|c|c|c|c|c|c|c|c|c|}
\hline Características & Definição da Norma & Critérios & $\begin{array}{l}\mathrm{N}^{\circ} \\
\text { Critérios } \\
\text { Totais }\end{array}$ & $\begin{array}{l}\mathbf{N}^{\circ} \\
\text { Critérios } \\
\text { Avaliados }\end{array}$ & $\begin{array}{l}\mathrm{N}^{\circ} \\
\text { Critérios } \\
\text { Excluídos }\end{array}$ & $\begin{array}{l}\mathbf{N}^{\circ} \\
\text { Critérios } \\
\text { Disp. }\end{array}$ & $\begin{array}{l}\mathrm{N}^{\circ} \\
\text { Critérios } \\
\text { Acep. }\end{array}$ & $\begin{array}{l}\mathrm{N}^{\circ} \\
\text { Critérios } \\
\text { Viab. }\end{array}$ \\
\hline $\begin{array}{l}\text { Melhoramento da } \\
\text { qualidade dos } \\
\text { dados e do serviço }\end{array}$ & $\begin{array}{l}\text { Norma 7. O estabelecimento de saúde recolhe, analisa e } \\
\text { emprega os dados sobre a utilização dos serviços e a qualidade } \\
\text { da atenção, desagregados por idade e sexo, com a finalidade } \\
\text { de respaldar o melhoramento da qualidade. O pessoal do } \\
\text { estabelecimento recebe apoio em favor de sua participação no } \\
\text { progresso contínuo da qualidade. }\end{array}$ & $59-70$ & 12 & 11 & 1 & 5 & 6 & 0 \\
\hline $\begin{array}{ll}\begin{array}{l}\text { Participação } \\
\text { adolescentes }\end{array} & \text { dos }\end{array}$ & $\begin{array}{l}\text { Norma 8. Os adolescentes devem participar no planejamento, } \\
\text { no seguimento e na avaliação dos serviços de saúde e nas } \\
\text { decisões relacionadas com a atenção de saúde que recebem e } \\
\text { também em alguns aspectos da prestação de serviços. }\end{array}$ & $71-79$ & 9 & 8 & 1 & 0 & 8 & 0 \\
\hline & & TOTAL & 79 & 75 & 4 & 35 & 38 & 2 \\
\hline
\end{tabular}

Fonte: A partir de OPAS/OMS, 2016 (p.4) (16).

TOTAL

Disp. $=$ Disponibilidade; Acep. $=$ Aceitabilidade Viab. $=$ Viabilidade Financeira

* A prestação de serviços no estabelecimento deve estar vinculada, segundo corresponda, à atenção que se oferta no nível dos centros de referência, nas escolas e em outros entornos comunitários 


\section{RESULTADOS}

Dos 35 profissionais de saúde entrevistados, 18 (51,4\%) eram de Mariño, 6 (17,1\%) de Península de Macanao e $11(31,4 \%)$ de Tubores. A maioria era do sexo feminino $(77,1 \%)$ e médicos $(85,7 \%)$. Os profissionais com menos de um ano no estabelecimento de saúde representaram 54,3\% (Tabela 1).

Foram entrevistados 246 adolescentes, 136 (55,3\%) de Mariño, 49 (19,1\%) de Península de Macanao e $61(24,8 \%)$ de Tubores. A metade era do sexo feminino, 49,2\% do sexo masculino e $2(0,8 \%)$ declararam ser homossexuais. O grupo etário com maior participação foi o de 12-14 anos com 53,3\%, seguido do de 15-19 anos com 43,1\%. Do total, 52\% declarou que havia utilizado os SS nos últimos seis meses, enquanto que os outros $48 \%$ não. Apenas 14 $(5,7 \%)$ declararam estar convivendo com seu parceiro, dos quais 11 (79\%) eram do sexo feminino. Do total, $89 \%$ eram estudantes e 5\% trabalhavam. Quase $100 \%$ dos entrevistados moravam com a família e $42 \%$ moravam com 5 ou mais pessoas (Tabela 2).

TABELA 1 -. Perfil dos profissionais e adolescentes entrevistados por municípios de Nueva Esparta, Venezuela, 2017

\begin{tabular}{|c|c|c|c|c|c|c|c|}
\hline \multicolumn{8}{|c|}{ Perfil dos profissionais entrevistados } \\
\hline \multirow{3}{*}{ Variável } & \multicolumn{5}{|c|}{ Municípios } & \multirow{2}{*}{\multicolumn{2}{|c|}{ Total geral }} \\
\hline & \multicolumn{2}{|c|}{ Mariño } & \multicolumn{2}{|c|}{$\begin{array}{l}\text { Península de } \\
\text { Macanao }\end{array}$} & Tubores & & \\
\hline & $\mathbf{n}$ & $\%$ & $\mathbf{n}$ & $\%$ & n $\%$ & n & $\%$ \\
\hline Sexo & 18 & 51,4 & 6 & 17,1 & 1131,4 & 35 & 100,0 \\
\hline Feminino & 13 & 37,1 & 4 & 11,4 & 1028,6 & 27 & 77,1 \\
\hline Masculino & 5 & 14,3 & 2 & 5,7 & $1 \quad 2,9$ & 8 & 22,9 \\
\hline Profissão & 18 & 51,4 & 6 & 17,1 & 1131,4 & 35 & 100,0 \\
\hline Médico & 17 & 48,6 & 4 & 11,4 & $9 \quad 25,7$ & 30 & 85,7 \\
\hline Enfermeira & 1 & 2,9 & 1 & 2,9 & 2,9 & 3 & 8,6 \\
\hline \multicolumn{8}{|c|}{ Técnico Superior Universitário em } \\
\hline Anos no estabelecimento & 18 & 51,4 & 6 & 17,1 & 1131,4 & 35 & 100,0 \\
\hline Menos de um ano & 10 & 28,6 & 3 & 8,6 & $6 \quad 17,1$ & 19 & 54,3 \\
\hline $1-5$ anos & 6 & 17,1 & 2 & 5,7 & $5 \quad 14,3$ & 13 & 37,1 \\
\hline Mais de 5 anos & 2 & 5,7 & 1 & 2,9 & 0,0 & 3 & 8,6 \\
\hline Mínimo-Máximo & \multicolumn{2}{|c|}{$0-21$} & \multicolumn{2}{|c|}{$0-19$} & $0-5$ & \multicolumn{2}{|c|}{$0-21$} \\
\hline Anos de conclusão de estudos & & 51,4 & 6 & 17,1 & 1131,4 & 35 & 100,0 \\
\hline Menos de um ano & 6 & 17,1 & & 0,0 & 25,7 & 8 & 22,9 \\
\hline $1-5$ anos & 8 & 22,9 & 5 & 14,3 & 617,1 & 19 & 54,3 \\
\hline Mais de 5 anos & & 11,4 & 1 & 2,9 & 38,6 & 8 & 22,9 \\
\hline Mínimo-Máximo & \multicolumn{2}{|c|}{$1-28$} & \multicolumn{2}{|c|}{$1-22$} & $1-10$ & \multicolumn{2}{|c|}{$1-28$} \\
\hline \multicolumn{8}{|c|}{ Perfil dos adolescentes entrevistados } \\
\hline Sexo & 136 & 55,3 & 49 & 19,1 & 6124,8 & 246 & 100,0 \\
\hline Feminino & 63 & 25,6 & 26 & 10,6 & 3413,8 & 123 & 50,0 \\
\hline
\end{tabular}

P2P \& INOVAÇÃO, Rio de Janeiro, v. 6 n. 1, Ed. Especial, p.101-125, 2019. 


\begin{tabular}{|c|c|c|c|c|c|c|c|c|}
\hline Masculino & 73 & 29,7 & 21 & 8,5 & 27 & 11,0 & 121 & 49,2 \\
\hline Homossexual & & & 2 & & & & 2 & 0,8 \\
\hline Idade & 136 & 55,3 & 49 & 19,9 & 61 & 24,8 & 246 & 100,0 \\
\hline $10-11$ & 2 & 0,8 & 5 & 2,0 & 2 & 0,8 & 9 & 3,7 \\
\hline $12-14$ & 78 & 31,7 & 19 & 7,7 & 34 & 13,8 & 131 & 53,3 \\
\hline $15-19$ & 56 & 22,8 & 25 & 10,2 & 25 & 10,2 & 106 & 43,1 \\
\hline $\begin{array}{l}\text { Nos últimos } 6 \text { meses, visitou algum } \\
\text { estabelecimento de saúde ou algum } \\
\text { profissional da medicina por ter problemas } \\
\text { de saúde? }\end{array}$ & 136 & 55,3 & 49 & 19,9 & 61 & 24,8 & 246 & 100,0 \\
\hline Sim & 62 & 25,2 & 30 & 12,2 & 35 & 14,2 & 127 & 51,6 \\
\hline Não & 74 & 30,1 & 19 & 7,7 & 26 & 10,6 & 119 & 48,4 \\
\hline Religião & 136 & 55,3 & 49 & 19,9 & 61 & 24,8 & 246 & 100,0 \\
\hline Católica & 89 & 36,2 & 29 & 11,8 & 47 & 19,1 & 165 & 67,1 \\
\hline Cristiana & 8 & 3,3 & & & 1 & 0,4 & 9 & 3,7 \\
\hline Muçulmana & & & & & 3 & 1,2 & 3 & 1,2 \\
\hline Outra & 2 & 0,8 & 2 & 0,8 & 1 & 0,4 & 5 & 2,0 \\
\hline Nenhuma & 37 & 15,0 & 18 & 7,3 & 9 & 3,7 & 64 & 26,0 \\
\hline Estado Civil atual & 136 & 55,3 & 49 & 19,9 & 61 & 24,8 & 246 & 100,0 \\
\hline Solteiro(a) & 128 & 52,0 & 45 & 18,3 & 59 & 24,0 & 232 & 94,3 \\
\hline Convivência de casal & 8 & 3,3 & 4 & 1,6 & 2 & 0,8 & 14 & 5,7 \\
\hline Nível de educação mais alto & 136 & 55,3 & 49 & 19,9 & 61 & 24,8 & 246 & 100,0 \\
\hline Educação primária concluída & 15 & 6,1 & 6 & 2,4 & 6 & 2,4 & 27 & 11,0 \\
\hline Parte do ensino primário & 5 & 2,0 & 2 & 0,8 & & 0,0 & 7 & 2,8 \\
\hline Parte do ensino médio & 100 & 40,7 & 31 & 12,6 & 44 & 17,9 & 175 & 71,1 \\
\hline Ensino médio completo & 16 & 6,5 & 10 & 4,1 & 11 & 4,5 & 37 & 15,0 \\
\hline Ocupaçãa atual & 136 & 55,3 & 49 & 19,9 & 61 & 24,8 & 246 & 100,0 \\
\hline Estudante & 122 & 49,6 & 39 & 15,9 & 58 & 23,6 & 219 & 89,0 \\
\hline Do lar & 4 & 1,6 & 1 & 0,4 & 3 & 1,2 & 8 & 3,3 \\
\hline Trabalha & 4 & 1,6 & 5 & 2,0 & & & 9 & 3,7 \\
\hline Trabalha e estuda & 3 & 1,2 & 1 & 0,4 & & & 4 & 1,6 \\
\hline Nenhuma & 3 & 1,2 & 3 & 1,2 & & & 6 & 2,4 \\
\hline Com quem mora atualmente & 136 & 55,3 & 49 & 19,9 & 61 & 24,8 & 246 & 100,0 \\
\hline Com a família ou com os pais & 135 & 54,9 & 49 & 19,9 & 61 & 24,8 & 245 & 99,6 \\
\hline Sozinho & 1 & 0,4 & & & & & 1 & 0,4 \\
\hline Especifique & 136 & 55,3 & 49 & 19,9 & 61 & 24,8 & 246 & 100,0 \\
\hline Com mãe e pai & 82 & 33,3 & 30 & 12,2 & 35 & 14,2 & 147 & 59,8 \\
\hline Mãe & 35 & 14,2 & 10 & 4,1 & 14 & 5,7 & 59 & 24,0 \\
\hline Mãe e padrasto & 6 & 2,4 & 4 & 1,6 & 4 & 1,6 & 14 & 5,7 \\
\hline Pai & 3 & 1,2 & & 0,0 & 4 & 1,6 & 7 & 2,8 \\
\hline Sem os pais & 10 & 4,1 & 5 & 2,0 & 4 & 1,6 & 19 & 7,7 \\
\hline $\begin{array}{l}\text { Número de pessoas que moram com } \\
\text { você }\end{array}$ & 136 & 55,3 & 49 & 19,9 & 61 & 24,8 & 246 & 100,0 \\
\hline $0-4$ & 64 & 26,0 & 34 & 13,8 & 44 & 17,9 & 142 & 57,7 \\
\hline $5-10$ & 42 & 17,1 & 13 & 5,3 & 17 & 6,9 & 72 & 29,3 \\
\hline $11 \mathrm{e}$ mais & 30 & 12,2 & 2 & 0,8 & 0 & 0,0 & 32 & 13,0 \\
\hline
\end{tabular}

Fonte: A partir dos dados da pesquisa 
Nos resultados ponderados por normas e dimensões os três municípios têm um comportamento similar, com pontuações que variam entre 10,9 e 67,3\% (Tabela 1). Nos percentuais globais das normas, Península de Macanao foi o município melhor avaliado com $37,6 \%$, porém os valores por estabelecimentos flutuam entre 54,7\% (Península de Macanao) e 22,2\% (Mariño) (Tabela 1). As pontuações médias (>10\% - 40\%) indicam que nos três municípios há necessidade de uma melhoria importante. Existem vários estabelecimentos nos municípios de Península de Macanao e Tubores com percentuais entre $>40 \%$ e $80 \%$ (Tabela 1). As normas 1 e 5 obtiveram as maiores pontuações, enquanto as normas 2 e 8 foram as piores avaliadas. Os fatores relacionados com a disponibilidade afetam mais o cumprimento das normas 4 e 6 , os fatores de aceitabilidade as normas 2 e 8 e os fatores de viabilidade financeira a norma 6.

No que diz respeito à dimensão disponibilidade, Mariño obteve a pontuação máxima global com 38,4. Os valores por estabelecimentos variam entre 17,8 e 50,3 (ambos de Mariño). Um dos fatores que limita o acesso nesta dimensão é o ambiente dos estabelecimentos de saúde: mais de 50\% não contam com assentos/cadeiras adequados, água potável, nem com materiais informativos. Embora 31 (89\%) estabelecimentos tinha banheiro, estes não se encontravam disponíveis para os usuários pelas restrições de água, que geralmente chega a cada 3/7/10 dias; havia 6 estabelecimentos sem acesso à água. 
ARTIGO

? INOVAÇÃo

Tabela 2 - Resumo das pontuações ponderadas por regras e dimensões de acesso por municípios, Nueva Esparta, Venezuela, 2017

\begin{tabular}{|c|c|c|c|c|c|c|c|c|c|c|c|c|}
\hline \multirow{3}{*}{ Norma } & \multicolumn{12}{|c|}{ Municípios } \\
\hline & \multicolumn{4}{|c|}{ Mariño } & \multicolumn{4}{|c|}{ Península de Macanao } & \multicolumn{4}{|c|}{ Tubores } \\
\hline & Normas & Disp. & Acep. & Viab. & Normas & Disp. & Acep. & Viab. & Normas & Disp. & Acep. & Viab. \\
\hline $\begin{array}{l}1 \text { - Conhecimentos básicos sobre } \\
\text { saúde dos adolescentes }\end{array}$ & 45,6 & 35,8 & 65,2 & NA & 47,9 & 38,2 & 67,3 & NA & 43,1 & 36,7 & 56,0 & NA \\
\hline 2 - Apoio comunitário & 20,5 & 44,4 & 10,9 & NA & 25,0 & 50,0 & 15,0 & NA & 27,1 & 50,0 & 17,9 & NA \\
\hline $\begin{array}{l}3 \text { - Conjunto de serviços } \\
\text { apropriados }\end{array}$ & 44,4 & 44,4 & NA & NA & 47,2 & 47,2 & NA & NA & 35,9 & 35,9 & NA & NA \\
\hline $\begin{array}{l}4 \text { - Competência dos provedores } \\
\text { de atenção de saúde }\end{array}$ & 29,7 & 15,6 & 41,4 & NA & 39,1 & 17,6 & 57,1 & NA & 34,5 & 19,7 & 46,8 & NA \\
\hline $\begin{array}{l}5 \text { - Características dos } \\
\text { estabelecimentos }\end{array}$ & 42,6 & 43,6 & 39,5 & NA & 45,9 & 47,0 & 42,2 & NA & 49,2 & 50,9 & 43,5 & NA \\
\hline 6 - Equidade e não discriminação & 30,6 & 52,8 & 32,8 & 13,9 & 42,8 & 29,2 & 39,3 & 58,3 & 43,3 & 29,5 & 42,5 & 52,3 \\
\hline $\begin{array}{l}7 \text { - Melhoramento da qualidade } \\
\text { dos dados e do serviço }\end{array}$ & 23,8 & 32,3 & 16,8 & NA & 29,3 & 37,2 & 22,7 & NA & 30,2 & 37,9 & 23,7 & NA \\
\hline 8 - Participação dos adolescentes & 21,1 & NA & 21,1 & NA & 23,3 & NA & 23,3 & NA & 27,6 & NA & 27,6 & NA \\
\hline Total & 32,3 & 38,4 & 32,5 & 13,9 & 37,6 & 38,1 & 38,1 & 58,3 & 36,4 & 37,2 & 36,9 & 52,3 \\
\hline
\end{tabular}

FONTE: A partir dos dados da pesquisa

Disp. $=$ Disponibilidade; Acep. $=$ Aceitabilidade; Viab. $=$ Viabilidade Financeira; NA= Não se aplica

Pontuação $\leq 10 \%$

Pontuação de $>10 \%$ - $\leq 40 \%$

Pontuação de $>40 \%-\leq 80 \%$

Falta de cumprimento das normas

Pontuação $>80 \%$

Necessidade de uma melhoria importante

Necessidade de alguma melhoria

Cumprimento das normas

P2P \& INOVAÇÃO, Rio de Janeiro, v. 6 n. 1, Ed. Especial, p.101-125, 2019. 
Outro aspecto identificado foi a falta de medicamentos essenciais, anticoncepcionais orais e insumos em mais de $60 \%$ dos estabelecimentos. Além disso, $89 \%$ dos profissionais indicaram desconhecer o conjunto de serviços de informação, orientação, tratamento e atenção que se prestam aos adolescentes; 74\% afirmaram que não fazem a promoção dos SS para adolescentes, e quase $90 \%$ dos adolescentes entrevistados desconhece os SS que são oferecidos para eles.

Embora apenas $28 \%$ dos profissionais indicaram que os horários de atendimento não se adequam às necessidades dos adolescentes, os horários geralmente estão restringidos ao turno da manhã. Em vários estabelecimentos de difícil acesso (Tubores/Península de Macanao) os médicos vão uma ou duas vezes por semana, em horário restrito. Um dos serviços mais valorizados pelos adolescentes é o de odontologia, que não estava funcionando em alguns estabelecimentos.

Em relação à aceitabilidade, a Península de Macanao obteve a pontuação máxima global com 38,8. No entanto, há estabelecimentos com pontuações entre 12,3 (Mariño) e 43,2 (Península de Macanao). Nesta dimensão a obrigatoriedade de ir com o representante em 74\% dos estabelecimentos é um obstáculo para o acesso, em $56 \%$ dos casos de adolescentes que indicaram que lhes haviam negado os serviços no estabelecimento próximo ao lugar de residência, o motivo foi por ser menor de idade e não estar com um adulto. A entrega de preservativos é também condicionada à autorização dos pais. Sobre a confidencialidade e o sigilo, em 38\% dos casos de adolescentes que haviam acessado os SS, o médico lhe havia assegurado que a informação não seria compartilhada com ninguém sem seu consentimento. Entretanto, 96\% confirmou que a política de confidencialidade não estava visível. Em 100\% dos estabelecimentos analisados não havia informação exposta sobre esta temática.

A limitada participação dos adolescentes é outro ponto destacado. Dos adolescentes atendidos nos últimos 6 meses, $46 \%$ opinaram que não participaram nas decisões relacionadas com sua atenção. Do total de adolescentes entrevistados, a mais de $92 \%$ nunca lhes propuseram ajudar ao pessoal do estabelecimento no planejamento dos SS. Quase 50\% dos profissionais indicaram não ter recebido treinamento em temas relacionados ao atendimento aos adolescentes no primeiro nível e $42 \%$ afirmou que não existe um sistema que lhe permita formação profissional contínua sobre os cuidados de saúde dos adolescentes.

A viabilidade financeira foi a única dimensão que teve avaliação positiva (superior ao $50 \%$ nos municípios Península de Macanao e Tubores). No entanto, nesta dimensão apenas dois critérios relativos à ausência/presença de políticas e diretrizes sobre prestação de serviços gratuita ou acessível para adolescentes foram considerados. Na Venezuela e no estado Nueva 
Esparta a prestação dos SS públicos é gratuita, não há cobrança direta aos usuários. Contudo, profissionais e adolescentes destacaram o impacto da crise econômica no acesso a: medicamentos, métodos anticoncepcionais, exames laboratoriais, terapias, etc., porque dependem do pagamento direto das famílias.

A variabilidade dos resultados pode ser observada na tabela 2, quando analisados separadamente por normas e por dimensão. A aceitabilidade é a dimensão com o maior número de estabelecimentos com pontuações entre $>10 \%$ e $40 \%$. A viabilidade financeira é a única com estabelecimentos com pontuações de $10 \%$.

\section{DISCUSSÃO}

Os achados revelam que os fatores que influenciam o acesso dos adolescentes aos SS são similares aos encontrados em outros estudos (CHECA; TAPIA, 2016; ESPOSTI et al., 2015; HIRMAS ADAUY et al., 2013; WORLD HEALTH ORGANIZATION, 2017). A importância que os adolescentes tenham conhecimentos básicos sobre sua saúde e os SS, é destacada por vários autores (CHECA; TAPIA, 2016; KRUK et al., 2018; MINISTERIO DEL PODER POPULAR PARA LA SALUD, 2012; OMS, 2014), porque isto fortalecerá suas capacidades para compreender, capacitar-se e participar ativamente na busca de assessoria, na identificação de SS e de fatores protetores (OPS; OMS, 2016b). A disponibilidade de material informativo nos estabelecimentos, como o trabalho intersetorial com educação e outros sectores da sociedade civil, é fundamental para o âmbito da norma 1.

$\mathrm{Na}$ América Latina continuam vigentes os discursos que estigmatizam e invisibilizam os adolescentes ao caracterizá-los como seres incompletos, imaturos e inseguros de si mesmos, afetando-os de maneira diferenciada segundo o gênero (CHAVES, 2005; GARCÍA SUÁREZ; PARADA RICO, 2018). Tais concepções influenciam as práticas institucionais e geram regras onde os adultos (pais/tutores/representantes e outros membros da comunidade) são atores-chave que podem apoiar ou dificultar o acesso de adolescentes os SS e a preservativos/anticoncepcionais orais (CHECA; TAPIA, 2016; KRUK et al., 2018; OPS; OMS, 2016b). A exigência da presença do adulto na maioria dos estabelecimentos é uma das principais barreiras que impacta a norma 2 , relacionada com a aceitabilidade.

A adequação dos estabelecimentos do primeiro nível de atenção para responder às necessidades dos adolescentes foi uma estratégia aplicada em países como o Uruguai, que teve bons resultados (DEPARTMENT OF HEALTH OF LONDON, 2011; WORLD HEALTH ORGANIZATION, 2013). O acesso desta população melhorou utilizando a rede de 
estabelecimentos do primeiro nível de atenção, com alguns pontos de referência com atenção especializada para adolescentes.

As alterações incluíram aspectos relacionados com os critérios das normas 3, 4, 5 e 7: consultas regulares para que os adolescentes saudáveis entrem em contato com os EBS; ajuste dos SS de acordo com as necessidades dos adolescentes e sua articulação com os outros níveis da rede (sistemas de referência/contra-referência); fortalecimento do sistema de informação; e construção de capacidades para a atenção aos adolescentes, através de processos de capacitação dos EBS (DEPARTMENT OF HEALTH OF LONDON, 2011; WORLD HEALTH ORGANIZATION, 2013). Esta pode ser uma referência aplicável na Venezuela e em Nova Esparta especificamente, já que o país conta com um número importante de estabelecimentos e médicos (HEREDIA; ARTMANN, 2018).

A localização é outro fator que poderia facilitar o acesso, dado que a maioria dos estabelecimentos está localizada entre 1 e $5 \mathrm{~km}$ de distância das comunidades de sua área de responsabilidade (HEREDIA; ARTMANN, 2018) possibilitando a continuidade do contato entre utentes e prestadores, o reforço dos laços de confiança (MINISTERIO DEL PODER POPULAR PARA LA SALUD, 2013), a inclusão nas comunidades de temas relacionados com a saúde dos adolescentes, incomum em vários países de médio e baixo rendimento (OMS, 2014), bem como a participação de adolescentes nas SS e identificação e trabalho com grupos de adolescentes vulneráveis (normas 6 e 8 ).

Dois aspectos a serem melhorados destacam a alta rotatividade de recursos humanos, especialmente dos médicos (HEREDIA; ARTMANN, 2018), e que os EBS tenham o que necessitam (materiais, insumos, medicamentos) para atender à população (KRUK et al., 2018), em espaços e horários adequados (OPS; OMS, 2014).

A ausência ou não adequação de fatores da dimensão disponibilidade pode ocasionar custos para os utilizadores (THIEDE; AKWEONGO; DI MCLNTYRE, 2014). A vulnerabilidade dos adolescentes num contexto de crise econômica é maior, porque as possibilidades da família fazer a despesa são mais limitadas (ROA, 2018). Na Venezuela, embora as consultas não sejam cobradas, utilizar os SS exige um desembolso de recursos próprios (nos casos em que é possível) e, portanto, a perda de receita (ROA, 2018) que afeta a viabilidade financeira.

A promoção da saúde dos adolescentes a partir da abordagem dos direitos é outro elemento fundamental. Incluir nos estabelecimentos informações claras sobre: os direitos dos adolescentes à informação, à uma atitude isenta de preconceitos e à uma atenção respeitosa; a políticas de confidencialidade e sigilo; à participação dos adolescentes nas SS, entre outros, 
permite socializar essa informação com os atores-chave e envolvê-los (KRUK et al., 2018; OPS; OMS, 2016b).

Os diferentes padrões nos municípios/casos e as diferenças entre as dimensões, bem como com as normas é um ponto a considerar (Tabela 2). As áreas que apresentam vantagens em termos de disponibilidade, na aceitabilidade podem ser consideradas aquém. A viabilidade financeira requer a inclusão de outros critérios para ser analisada com maior profundidade.

$\mathrm{Na}$ Venezuela, assim como em outros sistemas de informação dos países de médio e baixo rendimento, há poucos dados sobre o acesso dos adolescentes às SS (KRUK et al., 2018). A utilização de diferentes fontes de informação (verificação direta, entrevista com profissionais/utilizadores) (OPS; OMS, 2016a), embora exijam um esforço na coleta e processamento dos dados, permite complementar os dados e detectar com maior precisão os nós críticos. As pontuações ponderadas são indicadores de fácil compreensão por parte das equipes de saúde e podem ser ajustados conforme as necessidades.

A possibilidade de calculá-los até o mínimo nível de desagregação (estabelecimento de saúde) diminui o mascaramento das desigualdades quando se utilizam valores médios e contribui para que as equipes estaduais possam fazer uma priorização equitativa para distribuir os recursos limitados. Por exemplo, Mariño tem uma percentagem global para as normas de 32,28. No entanto, há um estabelecimento com pontuações menores a $20 \%$ segundo normas e nas três dimensões que deveria ser priorizado.

\section{CONSIDERAÇÕES FINAIS}

Nos municípios analisados os principais fatores que influenciam o acesso estão relacionados com a escassa promoção dos SS e o desconhecimento destes por parte dos adolescentes; a obrigatoriedade de comparecer com pais ou representantes; pouca informação sobre a confidencialidade e o sigilo e a limitada participação dos adolescentes. As normas 2, 4 e 8 e a dimensão aceitabilidade são as mais afetadas.

Estabelecer a linha base por estabelecimento de saúde e municípios para cada norma (OPS; OMS, 2016d), identificando o peso de critérios relativos a cada dimensão do acesso é uma novidade que permite identificar os estabelecimentos onde existem restrições para priorizar intervenções políticas adequadas e equitativas (THIEDE; AKWEONGO; DI MCLNTYRE, 2014) (Tabela 1). Os avanços na aplicação de estratégias para melhorar o acesso dos adolescentes podem ser monitorados com pontuações ponderadas por norma/dimensão do 
acesso nos diferentes níveis de desagregação. Consideramos, contudo, que os métodos quantitativos devem ser combinados com os qualitativos para potencializar a análise do acesso.

O processo de análise do acesso através da avaliação das normas pode converter-se em um espaço de capacitação/reflexão onde atores-chave (profissionais e usuários) socializam suas visões sobre o acesso dos adolescentes aos SS e que possa facilitar a integração das equipes de diversos níveis de gestão. Ainda que o foco seja nos SS para adolescentes um número importante de critérios avaliados poderiam servir como proxy para conhecer os fatores que afetam o acesso da população aos demais serviços de saúde oferecidos na área de responsabilidade.

Os resultados do estudo permitiram mostrar um possível caminho para produzir informações sobre o acesso dos adolescentes aos serviços de saúde, utilizando a estrutura triangular e as dimensões propostas por Thiede et al (2014), assim como as alternativas para enfrentar os problemas da escassa informação sobre algumas dimensões.

O ineditismo destas contribuições reflete-se na incorporação de aspectos que são de relevância para o planejamento em saúde, tais como: (1) o interesse em conhecer as causas subjacentes das diferenças de utilização entre os grupos definidos; (2) a definição de acesso incorpora a ideia de capacitação para tomar decisões bem fundamentadas sobre o uso desses serviços; (3) a valorização da dimensão da aceitabilidade para identificar como através dos processos de interação entre os serviços de saúde e a população, se pode obstaculizar o facilitar o acesso; (4) a comunicação e a confiança como aspectos medulares (5) o reconhecimento da importância de utilizar métodos quantitativos e qualitativos para explorar plenamente os problemas do acesso aos serviços de saúde; (6) a incorporação da responsividade como indicador do desempenho da assistência à saúde. 


\section{REFERENCIAS}

AITKEN, J.-M.; THOMAS, D. Synthesis of final evaluation findings from the Nepal safer motherhood project. Kathmandu: Ministry of Health, Department for International Development, 2004.

CHAVES, M. Juventud negada y negativizada: representaciones y formaciones discursivas vigentes en la Argentina contemporánea. Ultima Década, Valparaíso, v. 13, n. 23, p. 9-32, 2005.

CHECA, S.; TAPIA, S. (Coord.). Derechos sexuales y reproductivos de los y las adolescentes: diálogos posibles entre los jóvenes y los servicios de salud. Buenos Aires: Sociedad Argentina de Pediatría, 2016. Disponível em: 〈http://bit.ly/2zuc7xt>. Acesso em: 29 ago. 2019.

CLIFTON, D.; HERVISH, A. The World's Youth: 2013 Data Sheet Population Reference Bureau, 2013. Disponível em: <https://www.prb.org/youth-datasheet-2013/>. Acesso em: 11 fev. 2019.

DEPARTMENT OF HEALTH OF LONDON. Quality criteria for young people friendly health services. Department of Health of London, 2011.

DONABEDIAN, A. Aspects of Medical Care Administration. Cambridge: Haward University Press, 1973.

DONABEDIAN, A. Evaluación de la calidad de la atención médica. Revista Calidad Asistencial, v. 16(1), p. 11-27, 2001.

ESPOSTI, C. D. D. et al. Representações sociais sobre o acesso e o cuidado pré-natal no Sistema Único de Saúde da Região Metropolitana da Grande Vitória, Espírito Santo. Saúde e Sociedade, São Paulo, v. 24, n. 3, p. 765-779, 2015.

GARCÍA SUÁREZ, C. I.; PARADA RICO, D. A. "Construcción de adolescencia": una concepción histórica y social inserta en las políticas públicas. Universitas Humanística, v. 85, n. 85, 29 jun. 2018.

GILSON, L. Aceitabilidade, confiança e equidade. In: MCINTYRE, D.; MOONEY, G. (Org.). Aspectos econômicos da equidade em saúde. Rio de Janeiro: Editora Fiocruz, 2014. p. 163190.

GOBIERNO DE CHILE; MINISTERIO DE SALUD. Servicios de salud integral, amigables y de calidad para adolescentes, con énfasis en salud sexual y reproductiva. Orientación técnica para la atención primaria de salud, 2017. Gobierno de Chile/ Ministerio de Salud, 2017. 
GOSTIN, L.; HODGE, J. The Domains of Responsiveness: a human rights analysis. World Health Organization, 2003. Disponível em: <http://www.who.int/iris/handle/10665/73926>. Acesso em: 19 fev. 2019.

HEREDIA-MARTÍNEZ, H.; ARTMANN, E. Criterios para la (re)distribución equitativa de los equipos básicos de salud en el nivel local en Venezuela. Cadernos de Saúde Pública, Rio de Janeiro, v. 34, n. 10, e00171117, 2018.

HIRMAS ADAUY, M. et al. Barreras y facilitadores de acceso a la atención de salud: una revisión sistemática cualitativa. Revista Panamericana de Salud Pública, v. 33, n. 3, p. 223 229, mar. 2013.

INSTITUTO NACIONAL DE ESTADÍSTICA. Proyecciones de Población. Instituto Nacional de Estadística, 2018.

INTERAGENCY TASK TEAM ON HIV; YOUNG PEOPLE. Guidance brief: HIV Interventions in the Health Sector for Young PeopleUnited Nations Population Fund, $2008 . \quad$ Disponível em: <https://www.who.int/maternal_child_adolescent/documents/iatt_hivandyoungpeople/en/>. Acesso em: 11 fev. 2019.

KRUK, M. E. et al. High-quality health systems in the sustainable development goals era: time for a revolution. The Lancet Global Health, Londres, v. 6, n. 11, p. e1196-e1252, 2018.

MINISTERIO DEL PODER POPULAR PARA LA SALUD. Fundamentos de las Normas de los Programas de Salud. Viceministerio de Redes de Salud Colectiva, Dirección General de Programas de Salud, MPPS, 2012.

MINISTERIO DEL PODER POPULAR PARA LA SALUD. Norma Oficial para la Atención Integral en Salud Sexual y Reproductiva. OPS/OMS, UNICEF, UNFPA, 2013.

OLIVER, A. Equity of access to health care: outlining the foundations for action. Journal of Epidemiology \& Community Health, v. 58, n. 8, p. 655-658, 1 ago. 2004.

OMS - ORGANIZACIÓN MUNDIAL DE LA SALUD. Salud para los adolescentes del mundo: una segunda oportunidad en la segunda década. Ginebra, 2014.

OMS; OPS; MINISTERIO DE SALUD, PRESIDENCIA DE LA NACIÓN. Evalaución de calidad. Una guía para la evaluación de los servicios de salud amigables para los adolescentes. OMS/OPS, 2009. Disponível em: <https://apps.who.int/iris/bitstream/handle/10665/85329/9789241598859_spa.pdf?sequence =1>. Acesso em: 22 fev. 2019. 
OPS - ORGANIZACIÓN PANAMERICANA DE LA SALUD; OMS - ORGANIZACIÓN MUNDIAL DE LA SALUD. Estrategia para el acceso universal a la salud y la cobertura universal de salud. Caracas, 2014.

OPS; OMS. Normas mundiales para mejorar la calidad de los servicios de atención de salud de los adolescentes. Guía de aplicación de un enfoque fundamentado en las normas para mejorar la calidad de los servicios de salud prestados a los adolescentes. Volumen 3: Herramientas para la realización de encuestas de calidad y de cobertura con el fn de obtener datos sobre el cumplimiento de las normas mundiales. OPS/OMS, 2016a.

OPS; OMS. Normas mundiales para mejorar la calidad de los servicios de atención de salud de los adolescentes. Guía de aplicación de un enfoque fundamentado en las normas para mejorar la calidad de los servicios de salud prestados a los adolescentes. . Volumen 1: Normas y criterios. OPS/OMS, 2016 b.

OPS; OMS. Normas mundiales para mejorar la calidad de los servicios de atención de salud de los adolescentes. Guía de aplicación de un enfoque fundamentado en las normas para mejorar la calidad de los servicios de salud prestados a los adolescentes. Volumen 2: Guía de aplicación. OPS/OMS, 2016c.

OPS; OMS. Normas mundiales para mejorar la calidad de los servicios de atención de salud de los adolescentes. Guía de aplicación de un enfoque fundamentado en las normas para mejorar la calidad de los servicios de salud prestados a los adolescentes. Volumen 4: Hojas de puntuación para el análisis de los datos. OPS/OMS, 2016d.

PATTON, G. C. et al. Next steps for adolescent health: a Lancet Commission. The Lancet, v. 383, n. 9915, p. 385-386, fev. 2014.

PENCHANSKY, R.; THOMAS, J. W. The Concept of Access: Definition and Relationship to Consumer Satisfaction. Medical Care, v. 19, n. 2, p. 127-140, fev. 1981.

PEREIRA, M. G. Epidemiologia teoria e prática. Rio de Janeiro: Guanabara Koogan, 2008.

ROA, A. C. Sistema de salud en Venezuela: ¿un paciente sin remedio? Cadernos de Saúde Pública, Rio de Janeiro, v. 34, n. 3, e00058517, 2018.

THIEDE, M.; AKWEONGO, P.; MCINTYRE, D. Explorando as dimensões do acesso. In: MCINTYRE, D.; MOONEY, G. (Org.). Aspectos econômicos da equidade em saúde. Rio de Janeiro: Editora Fiocruz, 2014. p. 137-161.

VAITSMAN, J.; ANDRADE, G. R. B. DE. Satisfação e responsividade: formas de medir a qualidade e a humanização da assistência à saúde. Ciência \& Saúde Coletiva, v. 10, n. 3, p. 599-613, set. 2005. 
VENEZUELA; DIRECCIÓN ESTADAL DE SALUD DEL ESTADO NUEVA ESPARTA. Base de Datos del Sistema de Información en Salud del estado Nueva Esparta. Venezuela y Dirección Estadal de Salud del estado Nueva Esparta, 2017.

VENEZUELA; MINISTERIO DEL PODER POPULAR PARA LA SALUD. Base de Datos del Sistema de Información en Salud. Venezuela y Ministerio del Poder Popular para la Salud, 2017.

WORLD HEALTH ORGANIZATION. GLOBAL Accelerated Action for the Health of Adolescents (AA-HA!): guidance to support country implementation. World Health Organization, Washington, DC, 24 set. 2018. Disponível em: <http://bit.ly/2NGachQ>. Acesso em: 29 ago. 2019.

WORLD HEALTH ORGANIZATION. Policy brief: HIV and adolescents - HIV testing and counselling, treatment and care for adolescents living with hiv, summary of key features and recommendations. World Health Organization, 2013. 